\title{
Breadth verses depth: the impact of tree structure on cultural influence
}

\author{
Rhodri L. Morris ${ }^{1,2}[0000-0002-9105-5378]$, \\ Liam D. Turner ${ }^{1,2[0000-0003-4877-5289]}$, \\ Roger M. Whitaker ${ }^{1,2[0000-0002-8473-1913]}$, and Cheryl Giammanco ${ }^{3}$ \\ 1 School of Computer Science \& Informatics, Cardiff University, Cardiff, UK \\ \{MorrisRL6, TurnerL9, WhitakerRM\}@cardiff.ac.uk \\ 2 Crime and Security Research Institute, Cardiff University, Cardiff, UK \\ 3 U.S. Army Combat Capabilities Development Command, \\ Army Research Laboratory, Aberdeen Proving Ground, MD, USA \\ cheryl.a.giammanco.civ@mail.mil
}

\begin{abstract}
Cultural spread in social networks and organisations is an important and longstanding issue. In this paper we assess this role of tree structures in facilitating cultural diversity. Cultural features are represented using abstract traits that are held by individual agents, which may transfer when neighbouring agents interact through the network structure. We use an agent-based model that incorporates both the combined social pressure and influence from an agent's neighbours. We perform a multivariate study where the number of features and traits representing culture are varied, alongside the breadth and depth of the tree. The results reveal interesting findings on cultural diversity. Increasing the number of features promotes strong convergence in flatter trees as compared to narrower and deeper trees. At the same time increasing features causes narrower deeper trees to show greater cultural pluralism while flatter trees instead show greater cultural homogenisation. We also find that in contrast to previous work, the polarisation between nodes does not rise steadily as the number of traits increase but under certain conditions may also fall. The results have implications for organisational structures - in particular for hierarchies where depth supports cultural divergence, while breadth promotes greater homogeneity, but with increased coordination overhead on the root nodes. These observations also support subsidiarity in deep organisational structures - it is not just a case of communication length promoting subsidiarity, but local cultural differences are more likely to be sustained within these structures.
\end{abstract}

Keywords: Agent-based Modelling · Organizational Structure · Cultural Diversity

\section{Introduction}

Beyond supporting communication, management and decision-making, organisational structures, both formal and informal, play an important role in shaping 
an organisation's culture [24]. Culture can be thought of as the values and norms held by the members of an organisation, or anything over which individuals can influence each other [2], such as beliefs or behaviours. Importantly, culture plays a role in affecting how organisations may function [10] and the organisation's identity $[23,15]$, alongside shaping the individuals that constitute them.

Interpersonal relationships play an important role in mediating cultural influence. One of the most fundamental structures underlying the relationships across an organisation is the tree - in other words a minimally connected sub-network that spans all nodes. Line management structures and hierarchies are common examples. Structures featuring tree-like branching are also not uncommon in groups where formal hierarchies are absent $[1,11,22]$.

Longstanding qualitative studies in the fields of business, sociology and psychology recognise the social implications of tall verses wide organisational structures $[4,14]$, primarily revealing findings concerning decision making from qualitative analysis. More recently, flatter organisational structures and 'holacracies' at companies such as Zappos and Valve have received much attention $[3,8,13$, $26]$ based on their alternative approaches to hierarchy. From a network perspective, wide tree structures typically offer a reduced path length for communication but can introduce bottlenecks through dependency on hubs. Conversely, deep structures circumvent this, but can introduce the problem of long communication chains that may impede activity when rapid responses are required. However, beyond communication and decision making, organisational structures also impact the culture of an organisation, as they represent how individuals may provide influence upon each other.

In this paper we investigate the specific role of tree structures in cultural propagation across a group, in particular looking at the shape of a tree, in terms of its breadth verses depth, and the resultant cultural diversity. Using an abstract representation of culture, our approach adopts agent-based modelling to understand how culture may become shared based on social influence. The model is composed of features held by agents, that can be shared through discrete traits. The underlying model [19] generalises the approach taken by Axelrod [2], and also incorporates combined influence from an agent's neighbours, based on social impact theory [17]. This allows us to determine the sensitivity of organisational structure to cultural propagation.

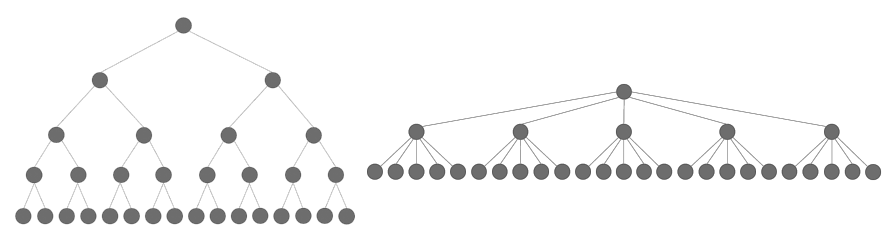

Fig. 1. A taller binary tree, $k=2, h=4$ (left), and a flatter tree $k=5, h=2$ (right). 


\subsection{Related Work}

Wide ranging literature supports the evolution of culture. From a computational perspective the work of Axelrod [2] provides a fundamental base model which has been extended in many directions. This approach introduces the idea that culture can be represented by a vector of features, where each feature takes a value from a set of discrete traits. Qualitative work [9] has proposed that in an organisational context, a large number of potential dimensions could be considered (e.g. 20). Axelrod's approach [2] involves agents randomly interacting in pairs over a lattice-based network structure with similarity between agents increasing the chance of a dissimilar feature being copied. This incorporates homophily, where more similar individuals have a greater disposition towards influencing each other [18].

There have been numerous generalisations of Axelrod's work, with two directions standing out in particular: firstly the structure over which cultural influence is performed $[16,25]$, and secondly the combined effect of influence from multiple neighbours $[12,19]$. In response, Axelrod's model has been examined on different network structures such as Erdős-Rényi random graphs [25], small-world and scale-free networks [16]. Equally compound influence has also been developed and examined, alongside agent-based models of culture represented through simple and complex contagion, often based on the susceptibility, infection and recovery (SIR) model of epidemic propagation [20,28].

Despite these contributions there has been limited consideration of hierarchies, either formal or informal, despite their prevalence in groups and organisations. Stocker et al [27] examined the effects of flat-vs-tall network structures on an opinion formation model, finding that flatter hierarchies have more fluctuations from consensus than tall. Nekovee et al [21] compared flat-vs-tall network structures and their effect on the spread of corruption through a network in an epidemic-based model. These models are typically limited in their consideration of culture, since they are limited to a binary representation [27] or variations on SIR-based models [21].

We contribute a new perspective by adopting an agent-based model [19] that considers the combined peer pressure upon the cultural features that an individual holds, while also incorporating the influence of homophily (i.e., similarity) between agents. This more realistically models the spreading of culture based on cumulative popularity of cultural features, and is applied to better understand the role tree structures in cultural propagation.

\section{Model}

We use the social-impact inspired model introduced in [19], an extension of Axelrod's model of Cultural Dissemination [2], as the basis for the analysis. The model assumes a set of $N$ agents (nodes) that are organised in a connected network structure (see 3). Each agent is assigned a vector of $F$ features $\left(\sigma_{1}, \sigma_{2}, \ldots, \sigma_{F}\right)$, each with a value from a set $T$ of $q$ possible traits, where $\sigma_{k}=1, \ldots, q$ for feature 
$k$. This defines an agent's culture, and agents can share some, none, or all traits. Edges between each pair of agents are weighted based on the similarity of their culture, using:

$$
\operatorname{sim}_{i, j}=\frac{1}{F} \sum_{k=1}^{F} \delta_{\sigma_{k}(i), \sigma_{k}(j)}
$$

where $\delta_{i, j}$ is Kronecker's delta.

At each iteration, a random agent $i$ is selected and is influenced by multiple neighbours simultaneously. Whether an agent adopts a trait is determined by the number of influencing agents having the trait in question and also the similarity of those influencers to the influenced agent. In this paper, we set the distance measure [19] for influencing neighbours to 1. For each feature $k$ where $k=$ $1, \ldots, F$, the selected agent calculates a trait score for each possible trait in $T$ and selects the value of $\alpha^{\prime} \in T$ that gives the maximal result. The trait score $t s_{\alpha, k, i}$ for node $i$, trait $\alpha$ and feature $k$ is calculated:

$$
t s_{\alpha, k, i}=\sum_{l=1}^{\max } \sum_{p^{l} \in P_{i}^{l}} w\left(p^{l}\right) \delta_{k, \alpha}\left(p^{l}\right)
$$

where $P_{i}^{l}$ as the set of all paths of length $l$ that end at $i ; p^{l} \in P_{i}^{l} ; w\left(p^{l}\right)$ is the product of all edge weights on $p^{l}$ and $\delta_{k, \alpha}\left(p^{l}\right)$ is a binary variable that is 1 if the source node of the path $p^{l}$ has $\alpha$ as its $k^{t h}$ feature. Thus the target agent $i$ copies the strongest trait $\alpha$ amongst its influencing neighbours.

\section{Methods}

We place agents in undirected balanced k-ary tree structures of height $h$ and branching factor $k$ and observe differences between wider, flatter trees (low $h$, higher $k$ ) and deeper, narrower trees (low $k$, higher $h$ ). Each tree has a root node at level 0 with $k$ children and $h$ levels of descendents (Figure 1). The values for $h$ and $k$ were chosen to maintain a similar number of agents across trees (see Table 1).

Table 1. Tree structure configurations as undirected networks

\begin{tabular}{|l|l|l|l|}
\hline Branching factor $(k)$ & Height $(h)$ & \# of Agents & \# of (undirected) Edges \\
\hline 2 & 9 & 1023 & 1022 \\
3 & 6 & 1093 & 1092 \\
4 & 5 & 1365 & 1364 \\
6 & 4 & 1555 & 1554 \\
10 & 3 & 1111 & 1110 \\
\hline
\end{tabular}



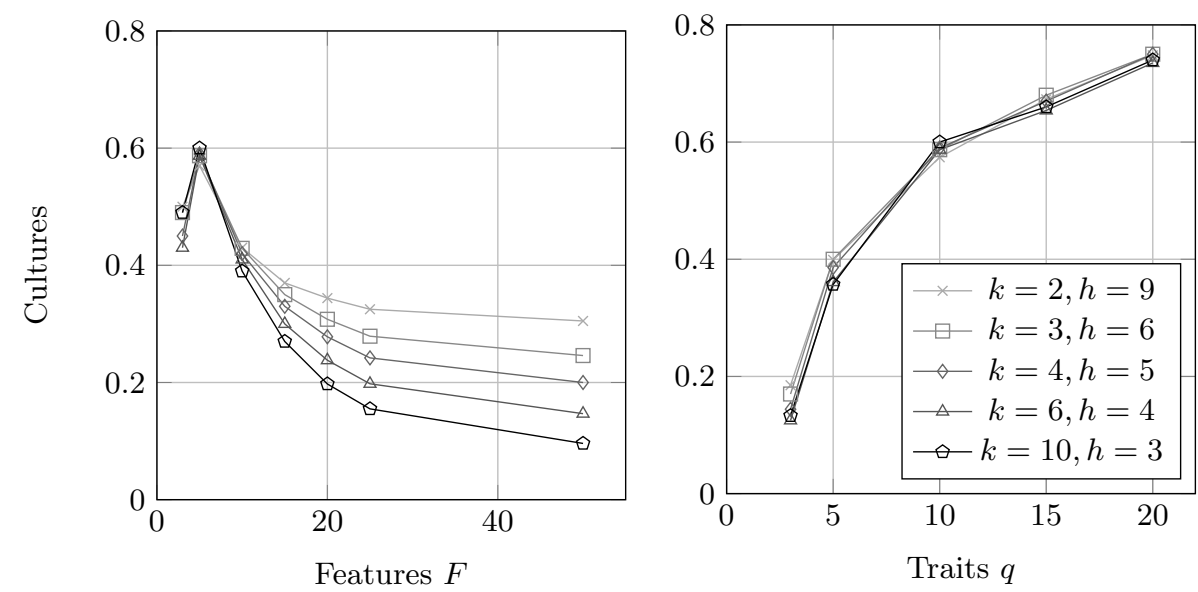

Fig. 2. Left: The number of cultures decreases as the size of the features vector, $F$, is increased. Deeper trees maintain a higher number of cultures than flatter trees. Note: $q=10$. Where $q=5$ and $q=15$ similar tall vs flat patterns are present. Right: The number of cultures increases as the number of possible traits, $q$, increases; however there is no discernible difference between different tree structures for $F=5$.

We vary the number of traits and features across the different tree shapes and observe the effect on cultural spreading behaviour. For each of the tree structures in table 1 we ran our model for $F=5, q=3,5,10,15,20,25,50$ and $q=10, F=3,5,10,15,25,50$. These parameters were chosen to exemplify the behaviours found in the model, between the states of complete mono-culture and complete polarisation. For each combination of parameters, we ran the simulation 20 times, each with a different random seed and random starting cultures. An additional 100 runs were made on each of $F=5 ; q=10,11,12,13,14,15$ to examine the behaviour discussed in section 4.1. For comparison, we also ran Axelrod's model [2] on similar trees and parameter sets as above, as well as a $32 \times 32$ square lattice (1024 agents).

Previous works $[5,6]$ based on [2] typically use the number of distinct cultures (i.e. unique trait combinations present at stabilisation) or largest cultural region size (largest area of contiguous identical agents) as metrics for cultural convergence and divergence. However, for our purposes these metrics do not fully illustrate the dynamics taking place because agents often hold traits from multiple cultures simultaneously and therefore cultures counted as distinct may in fact have many traits in common. Similarly, the largest region size may not adequately indicate the amount of fracture in other groups, or the amount of cultural overlap between this largest region and others. A dominant trait may persist across several otherwise different cultures. Consequently, we record the state of edges between nodes. Edges between two nodes with identical traits are considered homogenised, edges between two nodes with completely dissimilar traits are labelled polarised, and edges between nodes with a mix of common 

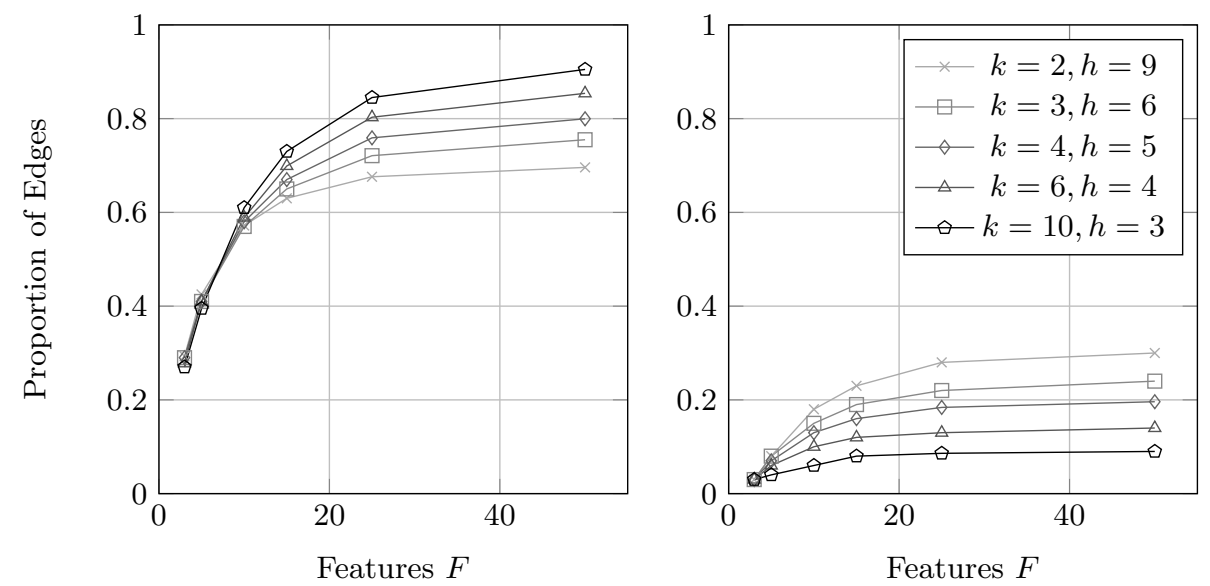

Fig. 3. Left: proportion of homogenised edges. Right: proportion of mixed edges. As the size of the features vector, $F$, increases, the number of polarised links drops. These links instead become homogenised or mixed. The amount of cultural mixing is greater for deeper trees (right) whereas flatter trees show greater convergence (left).

and differing traits are labelled mixed. At stabilisation, Axelrods model will never contain mixed edges; polarised edges may exist along borders of homogenised cultures, whereas in our model mixed edges can occur.

\section{Results}

As a baseline, we apply Axelrod's model [2] using tree topologies instead of the regular lattice structure. Interestingly the results show consistency with the patterns observed when applied to regular square lattice structures - we are unable to assert correlations between different tree shapes and the number of cultures emerging under the cultural model from [2]. This may serve as another example of how contagion dynamics differ when transmission is complex [7] rather than via a simple dyadic interaction. It also motivates the importance of considering combined influences in a cultural context, as presented in Section 2. We explore this further below.

\subsection{Polarised, homogenised and mixed links in a model of social influence}

We find that an increase in the number of features causes the proportion of polarised links to drop as a result of increased convergence. Consequently the proportion of homogenised and mixed links increase. The increase of homogenised links is greater in flatter trees than deep; the latter instead display a greater increase of mixed links (Figure 3). This is also reflected when counting the number of distinct cultures. While the decrease of cultures due to increased $F$ is 


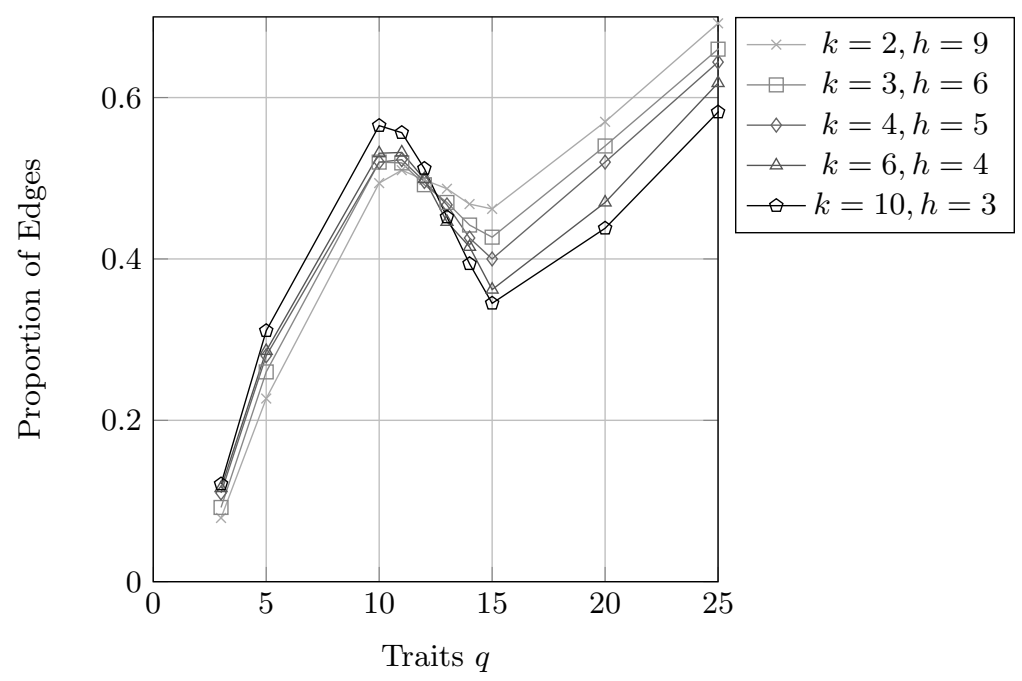

Fig. 4. Proportion of polarised edges when varying traits. As choice increases, an increase in polarisation is expected. However, between $10 \lesssim q \lesssim 15$ polarisation drops, and deeper trees become more polarised that flatter trees.

commonly observed $[2,19]$, the degree to which this decrease occurs is different in flatter trees than taller (Figure 2). Such a clear trend toward homogeneity in flatter tree structures may seem counter-intuitive if one envisions a typically tall hierarchy as more rigid and autocratic. However, the greater degree of intermediate 'hub' nodes, social reinforcement, and shorter paths of communication may aid convergence. This could have implications for the development of an organisational culture.

When the number of traits is varied, the proportion of homogenised links drops as agents have less chance of similarity. However, how the number of traits affects the proportion of mixed or polarised links is more nuanced (Figure 4). When the number of traits is small $(q \lesssim 10)$, polarisation rises as the number of traits increase. However, between $q \approx 10$ and $q \approx 15$ the proportion of polarised links drops, before rising again at higher trait levels. Furthermore, when $q \lesssim$ 10 flatter trees have a greater proportion of polarised links than deeper trees; however when $q \gtrsim 15$ the opposite is true.

In most previous works based on Axelrod's model (e.g. $[5,16])$ there has existed a positive correlation between the number of traits $q$ and the number of distinct cultures. While the number of cultures also increases in our model, this does not account for the degree to which cultures may overlap. Between $10 \lesssim q \lesssim 15$ the amount of polarisation between existing cultures drops, and cultural mixing increases (Figure 4). This behaviour is unusual, and has not been observed either in Axelrod's base model or when our model is run on lattice structures. The increase in otherwise different cultures sharing common traits also affects taller trees to a greater extent than it does flatter trees. The inversion 


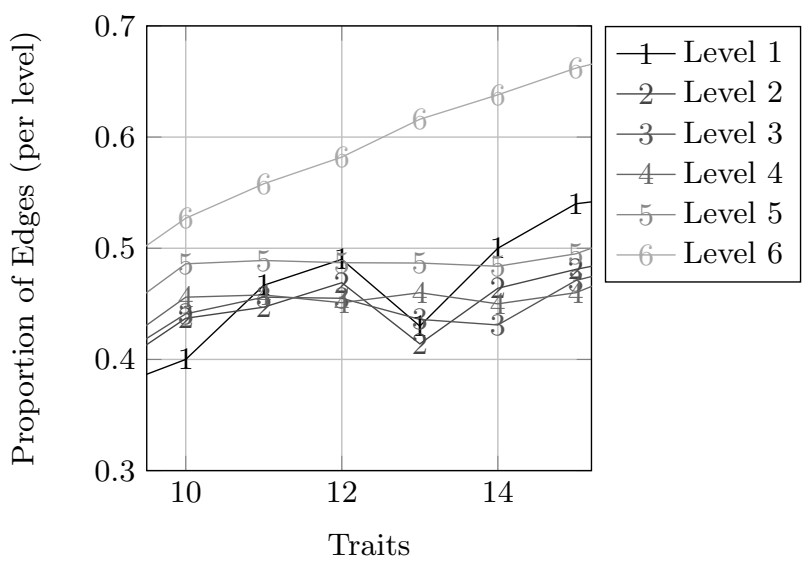

Fig. 5. Polarised edges at different levels of a $k=3, h=6$ tree. The higher in the tree an edge, the less likely it is to be polarised between $10 \lesssim q \lesssim 15$.

between $10 \lesssim q \lesssim 15$ suggests that whether a flatter tree structure exhibits more cultural mixing than tall depends on the amount of choices available per cultural feature.

\subsection{Polarisation at different tree levels}

To examine the dip in polarisation at $10 \lesssim q \lesssim 15$ we also record the proportion of polarised and mixed edges at each level in the tree, where edges at level $n$ are the edges between nodes at level $n$ and their parents. At the lowest level of the tree polarisation continues to climb between $10 \lesssim q \lesssim 15$. As we move up through the levels of the tree the rise in polarisation between $10 \lesssim q \lesssim 15$ is arrested, and at higher levels often reversed (Figure 5). As trees get flatter, this inversion of polarisation occurs at relatively lower levels and with a steeper decline.

To explain this further, we note that the nodes in the lowest level of these trees each only have a single connection - to their parent. The copying dynamics therefore dictate that they may only stabilise at a state either identical or dissimilar to their parent. As $q$ is increased, polarised links at this level always increase. Despite broader trees having a greater proportion of their nodes in this polarised lowest level, they show the greatest drop in polarisation between $10 \lesssim q \lesssim 15$. That the drop in polarisation occurs largely in the upper levels of the tree may be examined further by experiments on different network structures, such as scale-free or others with differing distributions of node degrees and centralities. 


\section{Conclusion}

In this paper we have applied a model of compound social influence, developed to capture the notion of peer pressure, to tree network structures of differing depth and breadth. We find that whether a tree is narrow and tall, or broad and flat, has a tangible effect on the amount of cultural convergence. In particular, as the number of features is increased flatter trees display greater cultural homogeneity than taller trees, which instead show greater cultural pluralism. When increasing the number of traits we also identify a point at which polarisation between nodes falls rather than rises, and taller trees become more polarised than flatter. Furthermore, some of these behaviours are particular to our model using compound influence as a copying dynamic, rather than dyadic interactions. This reinforces previous findings where complex contagions have resulted in different dissemination behaviours to simple contagions (e.g. [7]). The dynamics observed in these experiments have potential implications for the development of organisational cultures and how traits can propagate. Whether either subsidiarity or centralisation are sought, the shape of a hierarchy can have a notable effect on the spread of behaviours within.

Acknowledgement This research was sponsored by the U.S. Army Research Laboratory and the U.K. Ministry of Defence under Agreement Number W911NF16-3-0001. The views and conclusions contained in this document are those of the authors and should not be interpreted as representing the official policies, either expressed or implied, of the U.S. Army Research Laboratory, the U.S. Government, the U.K. Ministry of Defence or the U.K. Government. The U.S.

and U.K. Governments are authorized to reproduce and distribute reprints for Government purposes notwithstanding any copyright notation hereon.

\section{References}

1. Arazy, O., Nov, O., Ortega, F.: The [wikipedia] world is not flat: On the organizational structure of online production communities. In: 22nd European Conference on Information Systems, ECIS 2014. Association for Information Systems (2014)

2. Axelrod, R.: The dissemination of culture: A model with local convergence and global polarization. Journal of conflict resolution 41(2), 203-226 (1997)

3. Bernstein, E., Bunch, J., Canner, N., Lee, M.: Beyond the holacracy hype. Harvard business review 94(7), 13 (2016)

4. Carzo Jr, R., Yanouzas, J.N.: Effects of flat and tall organization structure. Administrative science quarterly pp. 178-191 (1969)

5. Castellano, C., Marsili, M., Vespignani, A.: Nonequilibrium phase transition in a model for social influence. Physical Review Letters 85(16), 3536 (2000)

6. Centola, D., Gonzalez-Avella, J.C., Eguiluz, V.M., San Miguel, M.: Homophily, cultural drift, and the co-evolution of cultural groups. Journal of Conflict Resolution 51(6), 905-929 (2007)

7. Centola, D., Macy, M.: Complex contagions and the weakness of long ties. American journal of Sociology 113(3), 702-734 (2007) 
8. Clegg, A.: Boss-less business is no workers paradise. Financial Times (Sep 2019), https://www.ft.com/content/34a86220-d639-11e9-8d46-8def889b4137

9. Delobbe, N., Haccoun, R.R., Vandenberghe, C.: Measuring core dimensions of organizational culture: A review of research and development of a new instrument. Unpublished manuscript, Universite catholique de Louvain, Belgium (2002)

10. Denison, D.R.: Corporate culture and organizational effectiveness. John Wiley \& Sons (1990)

11. Diefenbach, T., Sillince, J.A.: Formal and informal hierarchy in different types of organization. Organization studies 32(11), 1515-1537 (2011)

12. Flache, A., Macy, M.W.: Local convergence and global diversity: From interpersonal to social influence. Journal of Conflict Resolution 55(6), 970-995 (2011)

13. Foss, N.J., Dobrajska, M.: Valve's way: Wayward, visionary, or voguish? Journal of Organization Design 4(2), 12-15 (2015)

14. Hankinson, P.: An empirical study which compares the organisational structures of companies managing the worlds top 100 brands with those managing outsider brands. Journal of Product \& Brand Management (1999)

15. Hatch, M.J., Schultz, M.: Relations between organizational culture, identity and image. European Journal of marketing 31(5-6), 356-365 (1997)

16. Klemm, K., Eguíluz, V.M., Toral, R., San Miguel, M.: Nonequilibrium transitions in complex networks: A model of social interaction. Physical Review E 67(2), $026120(2003)$

17. Latané, B.: The psychology of social impact. American psychologist 36(4), 343 (1981)

18. McPherson, M., Smith-Lovin, L., Cook, J.M.: Birds of a feather: Homophily in social networks. Annual review of sociology 27(1), 415-444 (2001)

19. Morris, R., Turner, L., Whitaker, R., Giammanco, C.: The impact of peer pressure: Extending axelrod's model on cultural polarisation. In: 2019 IEEE International Conference on Cognitive Computing (ICCC). pp. 114-121. IEEE (2019)

20. Nekovee, M., Moreno, Y., Bianconi, G., Marsili, M.: Theory of rumour spreading in complex social networks. Physica A: Statistical Mechanics and its Applications 374(1), 457-470 (2007)

21. Nekovee, M., Pinto, J.: Modeling the impact of organization structure and whistleblowers on intra-organizational corruption contagion. Physica A: Statistical Mechanics and its Applications 522, 339-349 (2019)

22. Oedzes, J.J., Van der Vegt, G.S., Rink, F.A., Walter, F.: On the origins of informal hierarchy: The interactive role of formal leadership and task complexity. Journal of Organizational Behavior 40(3), 311-324 (2019)

23. Parker, M.: Organizational culture and identity: Unity and division at work. Sage (2000)

24. Schein, E.H.: Organizational culture., vol. 45. American Psychological Association (1990)

25. Singh, P., Sreenivasan, S., Szymanski, B.K., Korniss, G.: Accelerating consensus on coevolving networks: The effect of committed individuals. Physical Review E 85(4), 046104 (2012)

26. Spicer, A.: No bosses, no managers: the truth behind the 'flat hierarchy' facade. The Guardian (July 2018)

27. Stocker, R., Cornforth, D., Bossomaier, T.R.: Network structures and agreement in social network simulations. Journal of Artificial societies and social simulation $\mathbf{5}(4)(2002)$

28. Zhou, J., Liu, Z., Li, B.: Influence of network structure on rumor propagation. Physics Letters A 368(6), 458-463 (2007) 